\title{
Uso da inteligência artificial para predição de acidentes de trabalho com materiais biológicos em profissionais da saúde
}

\author{
Use of artificial intelligence for prediction of work accidents with biological risks in healthcare \\ professionals
}

Uso de la inteligencia artificial para la predicción de accidentes de trabajo con materiales biológicos en profesionales de la salud

\section{Resumo}

Este estudo buscou desenvolver um software que calcula a chance de o profissional de saúde ter zero, um, dois, três ou quatro ou mais acidentes com riscos biológicos. Para tal foram utilizados dados de 111 questionários de trabalhadores da saúde da atenção primária e pronto atendimento. O programa atingiu 95\% de acurácia no conjunto de treinamento $(n=88)$ e $74 \%$ no conjunto de teste $(n=23)$. As associações estatisticamente significantes, que contaram também com dados de 1.094 Comunicações de Acidente de Trabalho, foram maior abandono do acompanhamento por médicos após acidente com materiais biológicos na comparação com outros profissionais $(p=0.02)$, técnicos em enfermagem e maior prevalência de acidentes com materiais biológicos que outros profissionais $(\mathrm{p}<0.001)$, trabalhadores de pronto atendimento apresentam mais acidentes com materiais biológicos que profissionais da atenção primária $(\mathrm{p}<0.001)$ e aumento do abandono após acidente com materiais biológicos no triênio 2016-2018 na comparação com 2007-2009 $(\mathrm{p}<0.001)$.

Palavras-chave: Inteligência artificial; Acidentes ocupacionais; Saúde do trabalhador; Atenção primária à saúde.

\begin{abstract}
This study developed a software that calculates the chance of the health professional having zero, one, two, three or four or more accidents with biological hazards. Data from 111 questionnaires of health workers in primary and emergency care were used. The program achieved 95\% accuracy in the training set $(n=88)$ and $74 \%$ in the test set $(n=23)$. The statistically significant associations, which also relied on data from 1,094 work accident reports, were greater abandonment of follow-up by physicians after an accident with biological materials in comparison with other professionals $(p=0.02)$, nursing technicians and a higher prevalence of accidents with biological materials than other professionals $(p<0.001)$, emergency care workers have more accidents with biological materials than primary care professionals $(\mathrm{p}<0.001)$ and increased follow-up abandonment after an accident with biological materials in the 20162018 period compared to $2007-2009$ ( $\mathrm{p}<0.001)$.
\end{abstract}

Keywords: Artificial intelligence; Occupational accidents; Occupational health; Primary health care.

\section{Resumen}

El objetivo de este estudio es desarrollar un programa informático que calcule la probabilidad de que un profesional de salud tenga cero, uno, dos, tres o cuatro o más accidentes con riesgo biológico. Se utilizaron datos de 111 cuestionarios de trabajadores de la atención primaria y de urgencias. El programa alcanzó 95\% de precisión en el conjunto entrenamiento $(n=88)$ y $74 \%$ en el conjunto de prueba $(n=23)$. Las asociaciones estadísticamente significativas, que 
también incluyeron datos de 1.094 Comunicaciones de Accidentes de Trabajo, fueron el mayor abandono del seguimiento por parte de los médicos tras un accidente con materiales biológicos en comparación con otros profesionales $(\mathrm{p}=0,02)$, los técnicos de enfermería y mayor prevalencia de accidentes con materiales biológicos que otros profesionales $(\mathrm{p}<0,001)$, los trabajadores de urgencias presentan más accidentes con material biológico que los profesionales de atención primaria $(\mathrm{p}<0,001)$ y aumento del abandono tras accidente con material biológico en el trienio 2016-2018 respecto a 2007-2009 ( $<<0,001)$.

Palabras clave: Inteligencia artificial; Accidentes laborales; Salud laboral; Atención primaria.

\section{Introdução}

Profissionais de saúde podem ser expostos a várias rotas de transmissão de microrganismos realizando atividades laborais. As formas com maior chance de transmissão desses patógenos são via percutânea (por meio de objetos pontiagudos), membrana mucosa e pele não intacta (Ministério da Saúde, 2018; Souza-Borges, Ribeiro e Oliveira, 2014).

De acordo com informações do Ministério da Fazenda (2017) houveram 12.870 acidentes com exposição a doenças transmissíveis (CID Z20) no ano de 2015. Ocorreu crescimento expressivo na quantidade destas ocorrências na comparação com 2006, onde foram comunicados 1.230 acidentes deste tipo (Ministério da Fazenda, 2007).

Cerca de 60 patógenos tem potencial de transmissão por acidentes ocupacionais, como os vírus da Hepatite B (HBV), hepatite C (HCV) e da Imunodeficiência Humana (HIV), bactérias, parasitas e fungos (Souza-Borges, Ribeiro \& Oliveira, 2014; Tarantola et al, 2006). Após contato parenteral com objeto perfurocortante, o risco de infecção é $0,3 \%$ para HIV, 1,8\% para HBV e aproximadamente 30\% para HCV em indivíduos suscetíveis (Souza-Borges, Ribeiro \& Oliveira, 2014; Centers for Disease Control and Prevention, 2001).

Medidas profiláticas como acompanhamento clínico e laboratorial são essenciais para redução do risco de contaminação (Sardeiro et al, 2019). Muitos profissionais de saúde abandonam o acompanhamento após acidente com materiais biológicos ao não realizar as consultas e testes laboratoriais, potencialmente aumentando o risco de soroconversão (Sardeiro et al, 2019; Ministerio da Saúde, 2018; Almeida et al, 2015). A profilaxia pós exposição (PEP) contra HIV é considerada segura, pois de acordo com Thomas et al (2015), de 2772 pessoas que receberam a prescrição para a profilaxia dez pacientes (0,37\%) tratados foram infectados pelo HIV durante o período de estudo, contudo apenas um $(0,04 \%)$ caso pode ser atribuído a falha da PEP.

A inteligência artificial (AI) é um termo geral significando uso do computador com comportamento inteligente com pouca intervenção humana (Hamet \& Trembaly, 2017). Na área da medicina pode ser aplicado o conceito de Machine Learning pela utilização de algoritmos matemáticos que aprendem pela experiência (Hamet \& Trembaly, 2017).

Modelos de inteligência artificial são utilizados em diversas áreas da medicina. Existem diversas publicações com aplicações na área de detecção de câncer de mama (McKinney et al, 2020), radiologia e medicina nuclear (Hosny et al, 2018; Syed \& Zoga, 2018; Nensa, Demircioglu \& Rischpler, 2019), alvos terapêuticos (Theofilatos et al, 2014), cardiologia (Krittanawong et al, 2017; Johnson et al, 2018; Dey et al, 2020), medicina reprodutiva (WANG et al, 2019), medicina personalizada (Schork, 2019), medicina de emergência (Stewart, Sprivulis \& Dwivedi, 2018), nefrologia (Niel \& Bastard, 2019), oftalmologia (Kapoor, Walter \& Al-Aswad, 2018; Ting et al, 2019; Balyen, Peto, 2019), psiquiatria (Meyer-Lindenberg, 2018), dermatologia (Aggarwal, 2019), urologia (Suarez-Ibarrola et al, 2019) e oncologia (Shimizu \& Nakayama, 2020).

Existem exemplos na literatura de modelos criados utilizando inteligência artificial retreinados (Walsh, 2018; Cheng, 2019). Essa funcionalidade pode ser utilizada para ampliar o escopo de atuação e aumentar a acurácia destes modelos. 


\section{Metodologia}

\section{Desenho do Estudo}

Este estudo retrospectivo foi desenvolvido em duas fases: na primeira foram coletados dados de 1.094 acidentes de trabalho na vigilância epidemiológica (Tabela 1). Essa primeira fase foi essencial para descrever informações de todos os acidentes referentes ao sexo, idade, local de trabalho, acompanhamento após acidente e idade (Tabela 1).

A segunda fase foi realizada em seis unidades de Atenção Primária e duas Unidades de Pronto Atendimento na região Oeste do Paraná, Brasil. Um questionário com dezoito questões foi desenvolvido pelos pesquisadores para conseguir mais informações específicas dos profissionais de saúde, adicionando-as a aquelas coletadas na vigilância epidemiológica.

Os trabalhadores foram entrevistados e solicitados para preencherem o questionário durante o trabalho. Os pesquisadores coletaram os dados dos questionários entre os anos de 2018 e 2019 após aprovação pelo Comitê de Ética em Pesquisa da Universidade Federal do Paraná (UFPR) com número 93838318.6.000.0102. No total, a segunda fase teve 111 participantes ( $\mathrm{n}=111)$. Como base metodológica utilizou-se o trabalho de Pereira et al (2018).

\section{Análise dos dados}

Todos os dados foram armazenados no software Excel e as análises estatísticas foram realizadas no RStudio usando os testes Qui-Quadrado e T-Student. Razão de Chances (OR) foi usada para inferência estatística pela característica retrospectiva dos dados

\section{Modelo de Inteligência Artificial}

O modelo foi desenvolvido na linguagem de programação Python com a biblioteca Scikit-learn. Para classificar em 0,1,2,3 ou 4 ou mais acidentes foi aplicada a metodologia random forest com 100 estimadores em aprendizado supervisionado. Dados de 111 participantes foram distribuídos em dois grupos: um para treinar $(\mathrm{n}=88)$ o modelo e outro para testar $(\mathrm{n}=23)$ o modelo. Acurácia foi de $95 \%$ para o grupo de treino e $74 \%$ para o grupo de teste. Figuras 1 e 2 mostram a interface gráfica do programa na língua portuguesa e inglesa, respectivamente. 
Figura 1 e Figura 2: Versão em Português e Inglês do software.

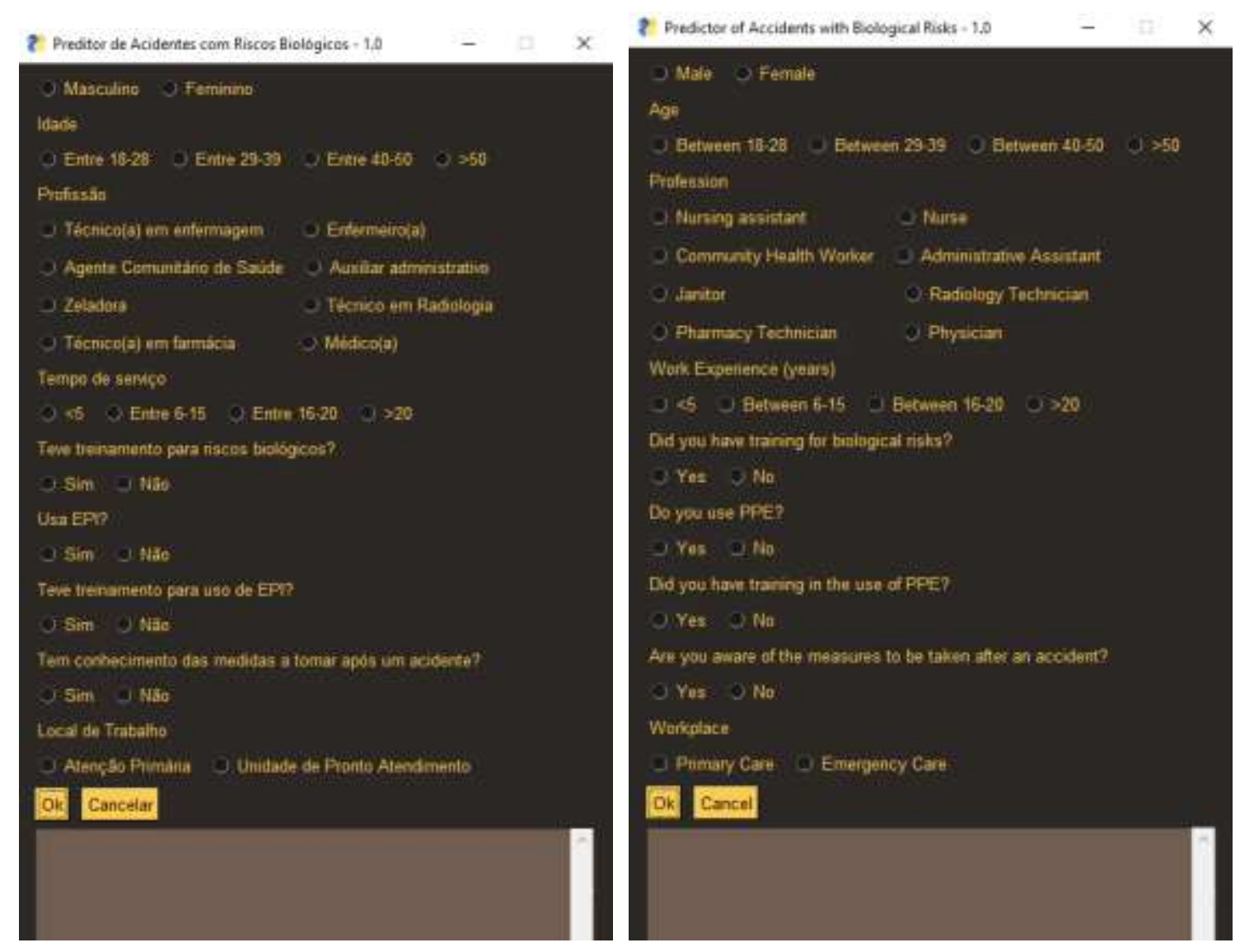

Fonte: Autores (2021)

\section{Resultados}

A Tabela 1 mostra dados colhidos na Vigilância Epidemiológica. A quantidade de acidentes, na comparação trienal, apresentou tendência de subida entre os períodos de 2007-2009 até 2013-2015, com leve queda em 2016-2018. Vários fatores, como subnotificação (Barbosa et al, 2017), podem estar envolvidos nessa aparente redução. A alta taxa de abandono do acompanhamento (59\%) (Tabela 2) ocorre em vários estudos da literatura (Sardeiro et al, 2019; Kon et al, 2011). 
Tabela 1: Informações disponíveis na Vigilância Epidemiológica (referentes a comunicações de acidentes de trabalho) $(\mathrm{n}=1094)$.

\begin{tabular}{ccc}
\hline Gênero & Acidentes & $\%$ \\
\hline Feminino & 935 & $85 \%$ \\
Masculino & 159 & $15 \%$
\end{tabular}

\section{Número de acidentes (ano)}

$\begin{array}{lcc}2007-2009 & 201 & 18 \% \\ 2010-2012 & 273 & 25 \% \\ 2013-2015 & 307 & 28 \% \\ 2016-2018 & 302 & 28 \% \\ 2019 \text { (parcial) } & 11 & 1 \%\end{array}$

\section{Acidentes por local de trabalho}

$\begin{array}{lcc}\text { Atenção Primária } & 133 & 12 \% \\ \text { Atenção Secundária } & 501 & 46 \% \\ \text { Atenção Terciária } & 369 & 34 \% \\ \text { Outros/Não Especificado } & 91 & 8 \%\end{array}$

\section{Acidentes por profissão}

\begin{tabular}{lcc} 
Assistente Administrativo & 5 & $0,5 \%$ \\
Agente Comunitário de Saúde & 1 & $0,1 \%$ \\
Médico & 40 & $4 \%$ \\
Técnico/Auxiliar de Enfermagem & 602 & $55 \%$ \\
Enfermeiro & 58 & $5 \%$ \\
Outros/Não Profissionais de Saúde & 388 & $35 \%$ \\
companhamento & & \\
Completou corretamente & 445 & $41 \%$ \\
Abandonou & 649 & $59 \%$ \\
cidentes por idade & & $39 \%$ \\
$18-28$ & 422 & $37 \%$ \\
$29-39$ & 401 & $19 \%$ \\
$40-50$ & 212 & $5 \%$ \\
$>50$ & 55 & $0,4 \%$ \\
Não especificado & 4 & \\
\hline
\end{tabular}

Fonte: Autores (2021).

Quase a metade dos acidentes ocorreu na atenção secundária (incluído unidades de pronto atendimento) (46\%), seguido pela atenção terciária (34\%) e por fim atenção primária (12\%) (Tabela 1). A hipótese de que profisssionais das unidades de pronto atendimento têm mais acidentes que aqueles da atenção primária foi considerada estatisticamente significante ( $<<0.001)$ (Tabela 3). 
A Tabela 2 resume os dados epidemiológicos coletados nos questionários. A maioria dos trabalhadores que preencheram os questionários e que tiveram acidentes relatados na vigilância epidemiológica são do sexo feminino. Além disso, a maioria dos profissionais têm idade entre 18-39 anos, o que reflete na maior proporção de acidentes nesses grupos etários. A maioria dos profissionais entrevistados trabalha na atenção primária (56\%) e cerca de $44 \%$ trabalha na unidade de pronto atendimento (Tabela 1 e 2$)$.

Tabela 2: Informação sobre participantes que completaram os questionários ( $\mathrm{n}=111)$

\begin{tabular}{lcc}
\hline Gênero & Participantes & \% \\
\hline Feminino & 82 & $74 \%$ \\
Masculino & 28 & $25 \%$ \\
Não especificado & 1 & $1 \%$ \\
Idade (anos) & & \\
$18-28$ & 21 & $19 \%$ \\
$29-39$ & 46 & $41 \%$ \\
$40-50$ & 29 & $26 \%$ \\
$>50$ & 15 & $14 \%$
\end{tabular}

\section{Profissão}

$\begin{array}{lcc}\text { Assistente Administrativo } & 8 & 7 \% \\ \text { Agente Comunitário de Saúde } & 35 & 32 \% \\ \text { Médico } & 15 & 14 \% \\ \text { Técnico/Auxiliar de Enfermagem } & 40 & 36 \% \\ \text { Enfermeiro } & 10 & 9 \% \\ \text { Outro } & 3 & 3 \%\end{array}$

\section{Experiência (anos)}

$\begin{array}{lcc}<6 & 54 & 49 \% \\ 6-15 & 47 & 42 \% \\ 16-20 & 4 & 4 \% \\ >20 & 6 & 5 \%\end{array}$

\section{Local de Trabalho}

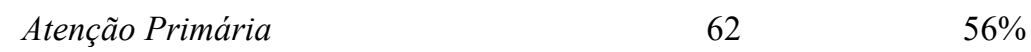

$\begin{array}{ll}\text { Unidade de Pronto Atendimento } & 49 \\ 44 \%\end{array}$

Fonte: Autores (2021).

A Tabela 3 mostra as hipóteses estatisticamente relevantes. Os dados do estudo mostraram que médicos abandonam mais acompanhamento que outros profissionais de saúde $(\mathrm{p}=0.02)$. Também foi observado aumento do abandono do acompanhamento após acidente entre os triênios 2016-2018 na comparação com 2007-2009 (p<0.001). Outra associação significativa foi entre mais acidentes com técnicos em enfermagem na comparação com outros profissionais ( $<<0.001)($ Tabela 3). Não houve diferença significativa associando sexo masculino ou feminino com maior predisposição a acidente com riscos biológicos $(p=0.37)$. 
Tabela 3: Razão de Chances e valor de p de hipóteses estatisticamente relevantes.

\begin{tabular}{|c|c|c|}
\hline Hipótese alternativa & Valor $p$ & $\begin{array}{c}\text { Razão de Chances } \\
\text { (IC 95\%) }\end{array}$ \\
\hline $\begin{array}{l}\text { Médicos abandonam mais acompanhamento após } \\
\text { acidente com materiais biológicos que outros } \\
\text { profissionais }\end{array}$ & 0.02 & $2.43(1.15-5.16)$ \\
\hline $\begin{array}{l}\text { Técnicos/Auxiliares em enfermagem têm mais } \\
\text { acidentes com materiais biológicos que outros } \\
\text { profissionais }\end{array}$ & $<0.001$ & $4.92(2.05-11.82)$ \\
\hline $\begin{array}{l}\text { Trabalhadores das unidades de pronto } \\
\text { atendimento têm mais acidentes com materiais } \\
\text { biológicos que profissionais da Atenção Primária }\end{array}$ & $<0.001$ & $5.21(2.11-12.84)$ \\
\hline $\begin{array}{l}\text { Aumento do abandono do acompanhamento após } \\
\text { acidente com materiais biológicos entre 2016-2018 } \\
\text { na comparação com 2007-2009 }\end{array}$ & $<0.001$ & $2.76(1.91-3.99)$ \\
\hline
\end{tabular}

Fonte: Autores (2021).

\section{Discussão}

Acidentes com materiais biológicos são um problema crescente nas instituições de saúde. O aumento estatisticamente significativo do abandono do acompanhamento entre os anos de 2016-2018, na comparação com 2007-2009 pode acarretar em aumento do risco de transmissão de patógenos como HIV, Hepatite B, Hepatite C aos profissionais de saúde (Sardeiro et al, 2019; Paiva \& Oliveira, 2011).

A tendência de crescimento dos acidentes entre os triênios 2007-2009 e 2013-2015 pode levar, além do risco de transmissão de patógenos aos trabalhadores da saúde, o aumento do sofrimento psíquico do profissional, pois reações como medo, culpa, desespero foram relatadas frente a essa situação inesperada (Magagnini, Rocha \& Ayres, 2011; Castanha, Machado \& Figueiredo, 2007).

A correlação entre trabalhadores da unidade de pronto atendimento e maior prevalência de exposição ocupacional a riscos biológicos $(\mathrm{p}<0.001)$ na comparação com profissionais da atenção primária pode ser explicada pela maior densidade tecnológica da atenção secundária ao realizar atendimentos de urgência e emergência (Erdmann et al, 2013) que leva aos profissionais a maior contato com materiais biológicos dos pacientes.

A maioria dos acidentes reportados ocorreu em trabalhadores com até 39 anos (Tabela 2). Essa informação é condizente com trabalho de Kon et al (2011) onde 43,2\% dos acidentes ocorreram na faixa etária dos 21-30 anos e 28,4\% entre 31-40 anos e em Silva et al (2009) onde 69,4\% dos acidentes ocorreram em profissionais entre 20 e 40 anos. Contudo, mais estudos são necessários para avaliar a hipótese de maior subnotificação destes acidentes em profissionais com mais de 40 anos.

A predominância de acidentes em mulheres (Tabela 2), consistente com dados na literatura é associada pela maior proporção de trabalhadoras do sexo feminino nas áreas da saúde (Tabela 1) (Kon et al, 2011). Contudo, não foi associado ao sexo feminino a maior prevalência de acidentes com materiais biológicos $(p=0.37)$.

Associação estatisticamente relevante $(\mathrm{p}<0.001)$ (Tabela 3) da elevada taxa de acidentes em auxiliares de enfermagem (55\%) (Tabela 2) pode ser explicada por representarem grande proporção dos trabalhadores na área da saúde (Tabela 1) (Chiodi, Marziale \& Robazzi, 2007) e terem contato direto com pacientes (Kon et al, 2011).

A utilização deste modelo de inteligência artificial pode auxiliar gestores da área da saúde e profissionais de saúde a avaliar quais medidas de proteção são mais adequadas considerando as especificidades de cada profissional de saúde, visando a redução da quantidade de acidentes com materiais biológicos. 


\section{Conclusão}

O presente trabalho apresentou dados epidemiológicos referentes aos profissionais de saúde de um município do Oeste do Paraná. Correlações estatisticamente relevantes, descritas na Tabela 3, como médicos abandonam follow-up após acidente mais que outros profissionais e técnicos em enfermagem têm mais acidentes que outros profissionais podem levar a políticas públicas focadas nestes trabalhadores.

O aumento do abandono do acompanhamento, englobando todos os profissionais, entre os anos 2016-2018 na comparação com 2007-2009 (Tabela 3) é preocupante por aumentar a possibilidade de contaminação do profissional de saúde, o que traz a necessidade de atividades como reuniões para conscientização e políticas que favoreçam o acompanhamento do profissional após acidente com material biológico.

O modelo de inteligência artificial foi treinado utilizando dados limitados a profissionais da atenção primária e unidades de pronto atendimento. Contudo, estudos utilizando inteligência artificial na área da medicina retreinaram modelos (Walsh et al, 2018; Cheng et al, 2019) para ampliar a amostra dos trabalhos. Dessa forma, futuros estudos podem estender o trabalho para outros centros de pesquisa, englobando atenção primária e unidades de pronto atendimento de outros municípios e também atenção terciária.

\section{Referências}

Aggarwal, L. P. (2019). Data augmentation in dermatology image recognition using machine learning. Skin Research and Technology, 25(6), 815-820. https://doi.org/10.1111/srt.12726

Almeida, M. C. M. de, Canini, S. R. M. da S., Reis, R. K., Toffano, S. E. M., Pereira, F. M. V., \& Gir, E. (2015). Clinical treatment adherence of health care workers and students exposed to potentially infectious biological material. Revista Da Escola de Enfermagem Da USP, 49(2), 0259-0264. https://doi.org/10.1590/S0080-623420150000200011

Balyen, L., \& Peto, T. (2019). Promising artificial intelligence-machine learning-deep learning algorithms in ophthalmology. Asia-Pacific Journal of Ophthalmology, 8(3), 264-272. https://doi.org/10.22608/APO.2018479

Barbosa, A. S. A. A., Do Amaral Diogo, G., Salotti, S. R. A., \& Silva, S. M. U. R. (2017). Subnotificação de acidente ocupacional com materiais biológicos entre profissionais de Enfermagem em um hospital público. Revista Brasileira de Medicina Do Trabalho, 15(1), 12-17. https://doi.org/10.5327/Z1679443520177034

Castanha, A. R., Machado, A. A., \& Figueiredo, M. A. de C. (2007). Conseqüências biopsicossociais do acidente ocupacional com material biológico potencialmente contaminado: perspectiva de pessoas do convívio íntimo do profissional da saúde. Rev. SBPH, 10(1), 65-84.

Centers for Disease Control and Prevention. (2001). Updated U.S. Public Health Service guidelines for the management of occupational exposures to HBV, $H C V$, and HIV and recommendations for postexposure prophylaxis.

Cheng, C. T., Ho, T. Y., Lee, T. Y., Chang, C. C., Chou, C. C., Chen, C. C., Chung, I. F., \& Liao, C. H. (2019). Application of a deep learning algorithm for detection and visualization of hip fractures on plain pelvic radiographs. European Radiology, 29(10), 5469-5477. https://doi.org/10.1007/s00330-019-06167-y

Chiodi, M. B., Marziale, M. H. P., \& Robazzi, M. L. do C. C. (2007). Occupational accidents involving biological material among public health workers. Revista Latino-Americana de Enfermagem, 15(4), 632-638. https://doi.org/10.1590/s0104-11692007000400017

Dey, D., Slomka, P. J., Leeson, P., Comaniciu, D., Shrestha, S., Sengupta, P. P., \& Marwick, T. H. (2019). Artificial Intelligence in Cardiovascular Imaging: JACC State-of-the-Art Review. Journal of the American College of Cardiology, 73(11), 1317-1335. https://doi.org/10.1016/j.jacc.2018.12.054

Erdmann, A. L., de Andrade, S. R., de Mello, A. L. S. F., \& Drago, L. C. (2013). A atenção secundária em saúde: Melhores práticas na rede de serviços. Revista Latino-Americana de Enfermagem, 21(SPL), 131-139. https://doi.org/10.1590/S0104-11692013000700017

Hamet, P., \& Tremblay, J. (2017). Artificial intelligence in medicine. Metabolism: Clinical and Experimental, 69, S36-S40. https://doi.org/10.1016/j.metabol.2017.01.011

Hosny, A., Parmar, C., Quackenbush, J., Schwartz, L. H., Aerts, H. J. W. L., \& Edu, H. H. (2018). Artificial intelligence in radiology HHS Public Access. Nat Rev Cancer, 18(8), 500-510. https://doi.org/10.1038/s41568-018-0016-5

Johnson, K. W., Torres Soto, J., Glicksberg, B. S., Shameer, K., Miotto, R., Ali, M., Ashley, E., \& Dudley, J. T. (2018). Artificial Intelligence in Cardiology. Journal of the American College of Cardiology, 71(23), 2668-2679. https://doi.org/10.1016/j.jacc.2018.03.521

Kapoor, R., Walters, S. P., \& Al-Aswad, L. A. (2019). The current state of artificial intelligence in ophthalmology. Survey of Ophthalmology, 64(2), 233-240. https://doi.org/10.1016/j.survophthal.2018.09.002

Kon, N. M., Soltoski, F., Reque, M., \& Do Amaral Lozovey, J. C. (2011). Acidentes de trabalho com material biológico em uma Unidade Sentinela: Casuística de 2.683 casos. Revista Brasileira de Medicina Do Trabalho, 9(1), 33-38. 
Krittanawong, C., Zhang, H. J., Wang, Z., Aydar, M., \& Kitai, T. (2017). Artificial Intelligence in Precision Cardiovascular Medicine. Journal of the American College of Cardiology, 69(21), 2657-2664. https://doi.org/10.1016/j.jacc.2017.03.571

Magagnini, M. A. M., Rocha, S. A., \& Ayres, J. A. (2011). O significado do acidente de trabalho com material biológico para os profissionais de enfermagem. Revista Gaúcha de Enfermagem, 32(2), 302-308. https://doi.org/10.1590/S1983-14472011000200013

McKinney, S. M., Sieniek, M., Godbole, V., Godwin, J., Antropova, N., Ashrafian, H., Back, T., Chesus, M., Corrado, G. C., Darzi, A., Etemadi, M., GarciaVicente, F., Gilbert, F. J., Halling-Brown, M., Hassabis, D., Jansen, S., Karthikesalingam, A., Kelly, C. J., King, D., \& Shetty, S. (2020). International evaluation of an AI system for breast cancer screening. Nature, 577(7788), 89-94. https://doi.org/10.1038/s41586-019-1799-6

Meyer-Lindenberg, A. (2018). Artificial intelligence in psychiatry-an overview. Nervenarzt, 89(8), 861-868. https://doi.org/10.1007/s00115-018-0557-6

Ministério da Fazenda. (2007). Anuário Estatístico de acidentes do trabalho.

Ministério da Fazenda. (2017). Anuário Estatístico de acidentes do trabalho.

Ministério da Saúde. (2018). Protocolo clínico e diretrizes terapêuticas para profilaxia pós-exposição (PEP) de risco à infecção pelo HIV, IST e Hepatites Virais.

Nensa, F., Demircioglu, A., \& Rischpler, C. (2019). Artificial intelligence in nuclear medicine. Journal of Nuclear Medicine, 60(9), 29S-37S. https://doi.org/10.2967/jnumed.118.220590

Niel, O., \& Bastard, P. (2019). Artificial Intelligence in Nephrology: Core Concepts, Clinical Applications, and Perspectives. American Journal of Kidney Diseases, 74(6), 803-810. https://doi.org/10.1053/j.ajkd.2019.05.020

Paiva, M. H. R. S., \& Oliveira, A. C. (2011). Fatores determinantes e condutas pós-acidente com material biológico entre profisisonais do atentimento préhospitalar. Revista Brasileira de Enfermagem, 64(2), 268-273. https://doi.org/10.1590/s0034-71672011000200008

Pereira A. S. et al. (2018). Metodologia da pesquisa científica. UFSM.

Sardeiro, T. L., de Souza, C. L., de Arvelos Salgado, T., Júnior, H. G., Neves, Z. C. P., \& Tipple, A. F. V. (2019). Work accidents with biological material: Factors associated with abandoning clinical and laboratory follow-up*. Revista Da Escola de Enfermagem, 53, 1-9. https://doi.org/10.1590/S1980$220 \times 2018029703516$

Schork, N. J. (2019). Artificial Intelligence and Personalized Medicine. Cancer Treatment and Research, 178, 265-283. https://doi.org/10.1007/978-3-03016391-4_11

Shimizu, H., \& Nakayama, K. I. (2020). Artificial intelligence in oncology. Cancer Science, 111(5), 1452-1460. https://doi.org/10.1111/cas.14377

Silva, J. A. da, Paula, V. S. de, Almeida, A. J. de, \& Villar, L. M. (2009). Investigação de acidentes biológicos entre profissionais de saúde. Escola Anna Nery, 13(3), 508-516. https://doi.org/10.1590/s1414-81452009000300008

Souza-Borges, F. R. F., Ribeiro, L. A., \& de Oliveira, L. C. M. (2014). Exposições ocupacionais a fluídos corporais e comportamentos em relação à sua prevenção e pós-exposição entre estudantes de medicina e de enfermagem de universidade pública Brasileira. Revista Do Instituto de Medicina Tropical de Sao Paulo, 56(2), 157-163. https://doi.org/10.1590/S0036-46652014000200012

Stewart, J., Sprivulis, P., \& Dwivedi, G. (2018). Artificial intelligence and machine learning in emergency medicine. EMA - Emergency Medicine Australasia, 30(6), 870-874. https://doi.org/10.1111/1742-6723.13145

Suarez-Ibarrola, R., Hein, S., Reis, G., Gratzke, C., \& Miernik, A. (2019). Current and future applications of machine and deep learning in urology: a review of the literature on urolithiasis, renal cell carcinoma, and bladder and prostate cancer. World Journal of Urology, Ml. https://doi.org/10.1007/s00345-019-03000-5

Syed, A. B., \& Zoga, A. C. (2018). Artificial Intelligence in Radiology: Current Technology and Future Directions. Seminars in Musculoskeletal Radiology, 22(5), 540-545. https://doi.org/10.1055/s-0038-1673383

Tarantola, A., Abiteboul, D., \& Rachline, A. (2006). Infection risks following accidental exposure to blood or body fluids in health care workers: A review of pathogens transmitted in published cases. American Journal of Infection Control, 34(6), 367-375. https://doi.org/10.1016/j.ajic.2004.11.011

Theofilatos, K., Pavlopoulou, N., Papasavvas, C., Likothanassis, S., Dimitrakopoulos, C., Georgopoulos, E., Moschopoulos, C., \& Mavroudi, S. (2015). Predicting protein complexes from weighted protein-protein interaction graphs with a novel unsupervised methodology: Evolutionary enhanced Markov clustering. Artificial Intelligence in Medicine, 63(3), 181-189. https://doi.org/10.1016/j.artmed.2014.12.012

Thomas, R., Galanakis, C., Vézina, S., Longpré, D., Boissonnault, M., Huchet, E., Charest, L., Murphy, D., Trottier, B., \& Machouf, N. (2015). Adherence to post-exposure prophylaxis (PEP) and incidence of HIV seroconversion in a major North American cohort. PLoS ONE, 10(11), 1-10. https://doi.org/10.1371/journal.pone.0142534

Ting, D. S. W., Pasquale, L. R., Peng, L., Campbell, J. P., Lee, A. Y., Raman, R., Tan, G. S. W., Schmetterer, L., Keane, P. A., \& Wong, T. Y. (2019). Artificial intelligence and deep learning in ophthalmology. British Journal of Ophthalmology, 103(2), 167-175. https://doi.org/10.1136/bjophthalmol-2018-313173

Walsh, S. L. F., Calandriello, L., Silva, M., \& Sverzellati, N. (2018). Deep learning for classifying fibrotic lung disease on high-resolution computed tomography: a case-cohort study. The Lancet Respiratory Medicine, 6(11), 837-845. https://doi.org/10.1016/S2213-2600(18)30286-8

Wang, R., Pan, W., Jin, L., Li, Y., Geng, Y., Gao, C., Chen, G., Wang, H., Ma, D., \& Liao, S. (2019). Artificial intelligence in reproductive medicine. Reproduction, 158(4), R139-R154. https://doi.org/10.1530/REP-18-0523 